\title{
Some Results of the Educational Experiment APIS (Cervantes Mission on Board ISS)
}

\author{
Pablo Fajardo · Sergio Barahona • Angel Sanz-Andres
}

\begin{abstract}
Some results of the analysis of the pictures taken along the performance of the Análisis de Propiedades Inerciales de Sólidos, Analysis of the Inertia Properties of Solid Bodies (APIS) experiment carried out in the Cervantes mission on board ISS, are presented. APIS was an educational experiment devoted to take advantage of the unique conditions of absence of relative gravity forces of a space platform such as ISS, to show some of the characteristics of the free rotational motion of a solid body, which are impossible to carry out on earth. This field of experimental research has application to aerospace engineering science (e.g. attitude control of spacecrafts), to astrophysical sciences (e.g. state of rotation and tumbling motions of asteroids) and to engineering education. To avoid the effect of the ambient atmosphere loads on the motion, the test body is placed inside a sphere, which reduces the effect of the aerodynamic forces to just friction. The drastic reduction of the effect of the surrounding air during the short duration of the experimental sequences allows us to compare the actual motion with the known solutions for the solid body rotation in vacuum. In this paper, some selected, relevant sequences of the sphere enclosing a body with a nominal cylindrical inertia tensor, put into rotation by the astronaut, are shown; the main problems to extract the information concerning the characteristic parameters of the motion are outlined, and some of the results obtained concerning the motion of the test probe are included, which show what seems
\end{abstract}

to be a curious and unexpected solution of the Euler equations for the solid body rotation in vacuum, without energy dissipation, when the angular momentum is almost perpendicular to the axisymmetry axis.

Keywords APIS · ISS · Solid body rotation • Aerospace engineering $\cdot$ Tumbling motion • Euler equations

\section{Introduction}

APIS comes from the Spanish acronym of "Análisis de Propiedades Inerciales de Sólidos" (Analysis of Inertial Properties of Solid Bodies). The general purpose of the experiment were mainly educational, aiming at showing the main characteristic of the free solid body rotation, which are of interest in engineering education, specially in aerospace engineering science, to explain among other things the attitude control of spacecrafts. In this regard, the classical example of the Explorer I unstable rotation, which is often used, dates back to 1958 (Wertz 1978). In addition to that, understanding this motion is also of interest in the astrophysics science field, applied to the study of the characteristics of asteroids. Light curve observations and other techniques are allowing improving the overall picture of rotational characteristics of asteroids and their relations to other asteroidal properties, as for instance, the relation between the rotation speed and the shape and internal structure of the asteroid. In this regard, one of the main questions is: are the asteroid agglomerates made up of numerous basic fragments, as rubble piles, held together by self-gravity, or are they monoliths with finite tensile strengths? It has been shown that a spin rate above some limiting value (depending of the shape and density) will result in the 
asteroid disintegrating unless it has some tensile strength (Pravec et al. 2002). In that way the rotation rates can give indications as to the origin and evolution of objects (Steel et al. 1997). In some cases, the rotation state is sometimes consequence of the disruption after collisions (Asphaug and Scheeres 1999) which can be traced back to the asteroid structure and the collision parameters. On the other hand, there is also interest in understanding the rotation or tumble rates of asteroids, as in the case that it should be necessary to divert an NEA found to be on collision course with the Earth, because the spin rate of the object is of considerable significance in the design of avoidance procedures.

The general purpose of the APIS experiment was a very wide one, aiming at to show several characteristics of the free motion: (a) the simple gyroscopic motion of an axisymmetric body, (b) the change of rotation axis due to energy dissipation, (c) misalignment between the initial angular velocity vector and principal inertia axes, namely with rotation around an axis almost perpendicular to the symmetry axis.

Several motions corresponding to the three types abovementioned have been recorded during the APIS experiment. The first type of motion (a) is conceptually trivial, and the reason for being included in the experiment, and the aim for its realization, was both just to demonstrate it and to check the system. The second type (b) was not clearly observed because the effect of the internal energy dissipation in the change of rotation axis orientation needs some time to develop, and during the experiments the rotating sphere jumped against the room walls (as a consequence of the drift motion of the centre of mass) before the effect could have been noticeable.

The case analyzed here, concerns only to the motion type $c$, and was carried out after the nominal sequences due to the initiative of the astronaut Pedro Duque, who took into account the lessons learned.

In what follows, the description of the problem is presented ("Description of the Problem"); the dynamics of the motion is described in terms of the evolution of the position of the principal inertia axis poles by using an asymptotic expansion of the classical solution of the Euler equations ("Motion of the Poles of the Principal Axes"); the image processing is summarized ("Experimental Images"), and the experimental results processing and analysis is presented (Experimental Results). Finally, conclusions are drawn in "Conclusions".

\section{Description of the Problem}

The test body consists of a threaded cylindrical aluminium bar which supports two coaxial short cylindrical

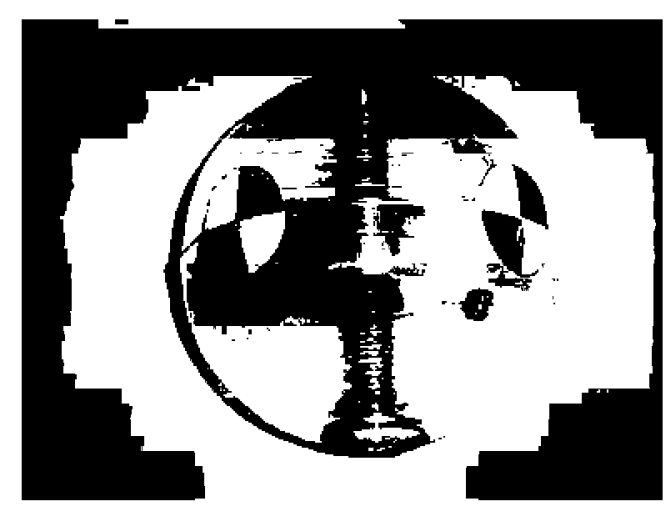

Fig. 1 One of the bodies tested during the APIS experiment, mounted inside a transparent sphere. In this picture the two disks are placed together at the sphere center

disks, perforated and also threaded so that they can be moved along the bar, as a nut on a bolt (see Fig. 1). The test body is mounted inside a sphere $(100 \mathrm{~mm}$ in diameter) so that aerodynamic loads are reduced just to the fluid friction effects, which can be neglected along short enough time evolution periods. In this case, as the sphere is otherwise free of external applied torque, the angular momentum can be taken as a constant. This very small effect, if any, cannot be confused with the effect of internal energy dissipation, which does preserve the angular momentum. Therefore, concerning the theoretical model of the motion, it can be assumed that we are dealing with the free motion of an axisymmetric body in vacuum. This problem, a classical one of the solid body mechanics, and widely known in the

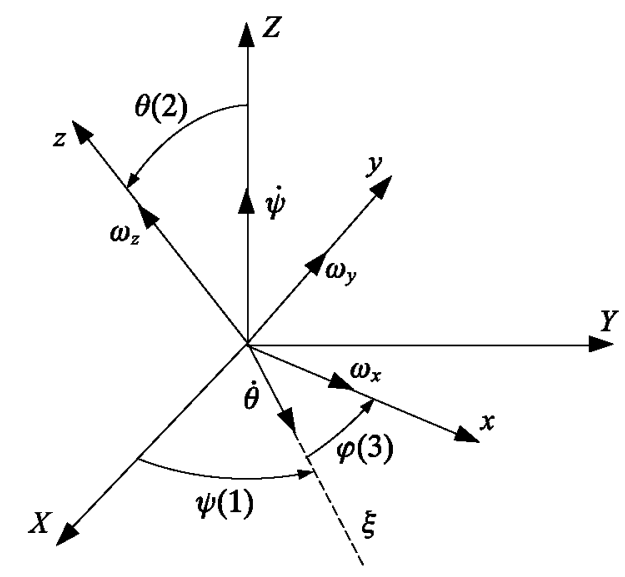

Fig. 2 Definition of the Euler angles. $\psi$, precession, $\theta$, nutation, $\varphi$, body spin. $X, Y, Z$, are the inertial coordinates system. $\omega_{x}, \omega_{y}, \omega_{z}$, rotation speed components. The figures indicate the order of the rotations to be performed to pass from reference frame $(X, Y, Z)$ to $(x, y, z)$ 


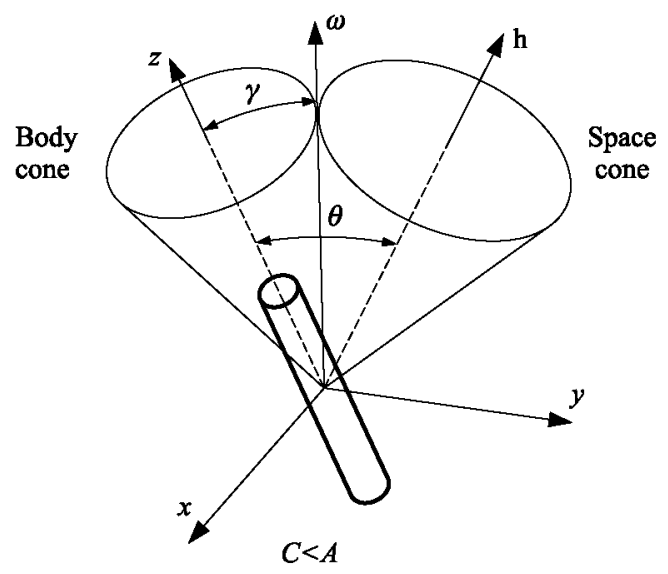

Fig. 3 Scheme of the Euler equations solution. Motion of the body represented as a cone (body cone) rolling on the space cone (fixed to inertial reference frame)

literature (Thomson 1986), is governed by the Euler equations for axisymmetric bodies

$$
\begin{aligned}
& A \dot{\omega}_{x}+(C-A) \omega_{y} \omega_{z}=0 \\
& A \dot{\omega}_{y}-(C-A) \omega_{x} \omega_{z}=0 \\
& C \dot{\omega}_{z}=0 \rightarrow \omega_{z}=n=c t e \\
& \omega_{x}=\dot{\psi} \sin \theta \sin \varphi \\
& \omega_{y}=\dot{\psi} \sin \theta \sin \varphi \\
& \omega_{z}=\dot{\varphi}+\dot{\psi} \cos \theta=n
\end{aligned}
$$

whose solution relevant to this study can be summarized as follows

$$
\begin{aligned}
\omega_{x y}^{2} & =\omega_{x}^{2}+\omega_{y}^{2}=c t e \\
\tan \theta & =\frac{A}{C} \frac{\omega_{x y}}{n}=c t e \\
\dot{\varphi} & =\frac{A-C}{A} n=c t e \\
\dot{\psi} & =\frac{C}{A} \frac{n}{\cos \theta}=c t e
\end{aligned}
$$

where $\omega_{x}, \omega_{y}, \omega_{z}$ are the rotation speed components in body axis, and $A, A$ and $C$ the inertia moments around body axes $x, y, z$, respectively (Fig. 2) which are the principal axes of inertia. The solution of this problem is very well know (Thomson 1986) and can be interpreted in terms of the motion of a cone (body cone, $x, y, z$ ) that rolls without sliding over a cone fixed in the inertial space (space cone $X, Y, Z$ ), as shown in Fig. 3. The inertial axis $Z$ orientation is chosen so that it is directed along the angular momentum $\mathbf{h}$. The axis of revolution of the body cone coincides with axis $z$. The axis of revolution of the space cone is the angular momentum $\mathbf{h}$, a constant which is the one that the body has when it is freed by the release mechanism (Fig. 4) and it coincides with the angular velocity vector if the body is made to rotate exactly around one of the principal inertia axis (Fig. 4a). The vertexes of both cones coincide at the centre of mass of the body (the sphere centre in this case). The contact line of both cones is the instantaneous rotation axis in the direction of the angular velocity vector.

In the case studied here (type c) the angular velocity vector at sphere release is almost perpendicular to the axisymmetric axis (see Fig. 4b).

The motion described in this way is easy to understand and can be easily observed in the case where the angle $\theta$ between $z$-axis and the angular momentum vector $\mathbf{h}$ is small, because motions like these resemble that of a spinning top. However, the description based on cone rotations has been found not to be of much help when trying to analyze and understand the images taken during the tests, where $\theta$ is large, and some additional analytical effort is needed to describe the motion in terms of what can be observed during the tests, where the camera point of view is placed close to the $Z$ axis (or h) direction. From the images taken during the test, data can be obtained concerning namely the motion of the principal inertia axes, which are labelled by using two-colours stickers as shown in Figs. 1 and 4. The colour code for the stickers in poles is $x^{+}-\mathrm{GW}, x^{-}$-GR,

Fig. 4 The sphere supported along a the $z$-axis (symmetry axis) and $\mathbf{b}$ along the $y$-axis (transversal axis) by the release mechanism, handled by the astronaut Pedro Duque. The disks are fully separated apart

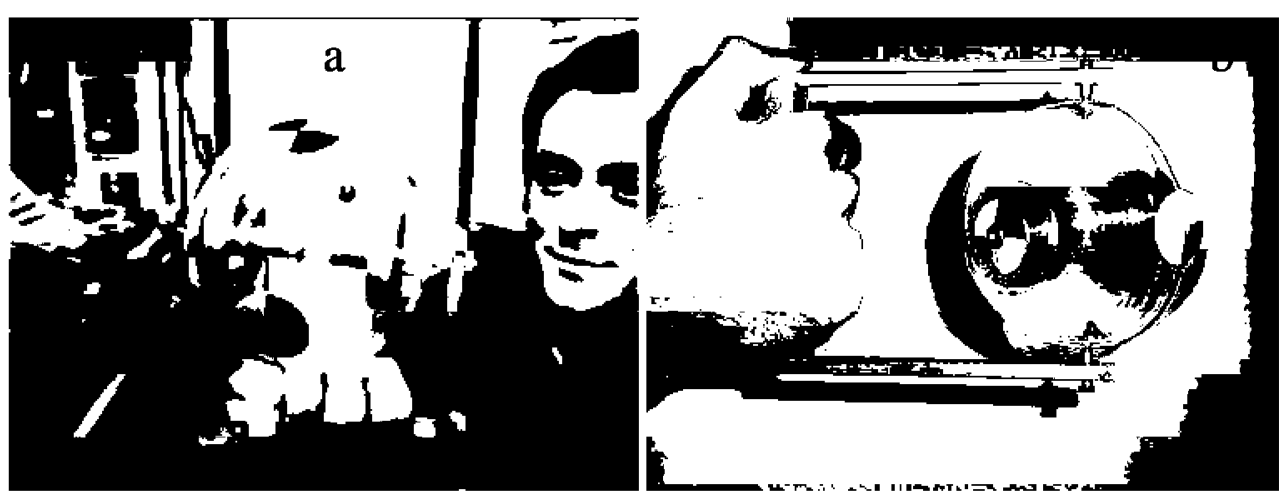


$y^{+}-\mathrm{KW}, y^{-}-\mathrm{KR}, z^{+}-\mathrm{BW}, z^{-}-\mathrm{WR}$, where $\mathrm{G}, \mathrm{W}, \mathrm{R}, \mathrm{K}, \mathrm{B}$ stand for green, white, red, black and blue, respectively.

The release mechanism (Fig. 4) is just a handle with two arms pressed together by the thumb against two supporting points attached to the sphere surface. In this position the sphere can spin freely around the axis defined by the two supporting points. When the thumb pressure is loosed the two arms separate, leaving the sphere freely rotating in space. The release should be done carefully to reduce at the minimum value the initial velocity of the centre of mass of the rotating body.

As above-mentioned, the comparison between experimental results and the Euler solution should be based on some description of the motion of these axes, which therefore becomes mandatory, and it is covered in the following section.

\section{Motion of the Poles of the Principal Axes}

The study of the motion of the principal axes poles is based on the formulation of axes transformation from body axes coordinates $x, y, z$ to inertial axes, $X, Y$, $Z$, for the free body rotation as follows (Thomson 1986, p. 37):

$$
\left[\begin{array}{l}
X \\
Y \\
Z
\end{array}\right]=\left[\begin{array}{ccc}
\cos \varphi \cos \psi-\sin \varphi \cos \theta \sin \psi & -\sin \varphi \cos \psi-\cos \varphi \cos \theta \sin \psi & \sin \theta \sin \psi \\
\cos \varphi \sin \psi+\sin \varphi \cos \theta \cos \psi & -\sin \varphi \sin \psi+\cos \varphi \cos \theta \cos \psi & -\sin \theta \cos \psi \\
\sin \theta \sin \varphi & \sin \theta \cos \varphi & \cos \theta
\end{array}\right]\left[\begin{array}{l}
x \\
y \\
z
\end{array}\right]=A\left[\begin{array}{l}
x \\
y \\
z
\end{array}\right]
$$

where $\theta(t), \varphi(t)$ and $\psi(t)$ are obtained from the motion equations. As deduced from Eq. 1c, in the free motion $\theta=c t e, \dot{\psi}=c t e ; \dot{\varphi}=c t e$, and therefore, $\varphi$ and $\psi$ grow in a linear way with time. Remember that the direction of projection $Z$ is assumed to be the direction of the angular momentum. The orientation of the reference frames is shown in Fig. 2. Let us consider the principal axes poles being placed on the unity radius sphere. Therefore, the trajectory in the inertial space of the point with the label showing the position of $z$-axis with coordinates $(0,0,1)$ is given by the transformation

$$
\left[\begin{array}{l}
X \\
Y \\
Z
\end{array}\right]=A\left[\begin{array}{l}
0 \\
0 \\
1
\end{array}\right]=\left[\begin{array}{c}
\sin \theta \sin \psi \\
-\sin \theta \cos \psi \\
\cos \theta
\end{array}\right]
$$

which is the last column of the transformation matrix $A$. The motion is assumed to be observed from a point placed far away along the $Z$ axis. Seen from this point, the position of $z$-axis pole is just its projection on the $X-Y$ plane, which can be denoted by the complex variable $\tau_{z}$

$$
\begin{aligned}
\tau_{z} & =X+\mathrm{i} Y=\sin \theta \sin \psi-\mathrm{i} \sin \theta \cos \psi \\
& =\sin \theta(\sin \psi-\mathrm{i} \cos \psi)=-\mathrm{i} \sin \theta e^{\mathrm{i} \psi}
\end{aligned}
$$

This expression shows that, in the free motion, $\tau_{z}$ describes a circumference with radius $\sin \theta$, which is swept at speed $\dot{\psi}=c t e$. In an analogous way the trajectory of $x$-axis $(1,0,0)$ is obtained from the first row of the transformation matrix $A$

$$
\begin{aligned}
& X=\cos \varphi \cos \psi-\sin \varphi \cos \theta \sin \psi \\
& Y=\cos \varphi \sin \psi+\sin \varphi \cos \theta \cos \psi \\
& Z=\sin \theta \sin \varphi
\end{aligned}
$$

As seen from $Z$ axis, the position of $x$-axis in the inertial reference frame projected on the plane $X-Y$ is given in complex variable form as $\tau_{x}$

$$
\begin{aligned}
\tau_{x}= & X+\mathrm{i} Y=\cos \varphi(\cos \psi+\mathrm{i} \sin \psi) \\
& +\sin \varphi \cos \theta(-\sin \psi+\mathrm{i} \cos \psi) \\
= & \cos \varphi e^{\mathrm{i} \psi /}+\sin \varphi \cos \theta \mathrm{i} e^{\mathrm{i} \varphi} \\
= & e^{\mathrm{i} \psi}(\cos \varphi+\mathrm{i} \sin \varphi \cos \theta)
\end{aligned}
$$

We can make use of the case of a free rotational motion without nutation, $\theta=0$, as a checking point. Actually, in this case, the position of $x$ axis is $\tau_{x}=e^{\mathrm{i} \psi /} e^{\mathrm{i} \varphi}=e^{\mathrm{i}(\varphi+\psi / t)}$, which is a circumference of unity radius swept at speed $\dot{\varphi}+\dot{\psi}=c t e$, as can be expected.

In an analogous way the trajectory of $y$ axis $(0,1,0)$ as seen from $Z$ axis is given in complex variable as

$$
\begin{aligned}
\tau_{y}= & X+\mathrm{i} Y=-\sin \varphi(\cos \psi+\mathrm{i} \sin \psi) \\
& +\cos \varphi \cos \theta(-\sin \psi+\mathrm{i} \cos \psi) \\
= & -\sin \varphi e^{\mathrm{i} \psi}+\cos \varphi \cos \theta \mathrm{i} e^{\mathrm{i} \varphi} \\
= & e^{\mathrm{i} \psi}(-\sin \varphi+\mathrm{i} \cos \varphi \cos \theta)
\end{aligned}
$$


An obvious check can be performed using also the case $\theta=0$. Again the classical result is obtained

$\tau_{y}=e^{\mathrm{i} \psi} \mathrm{i} e^{\mathrm{i} \varphi}=\mathrm{i} e^{\mathrm{i}(\varphi+\psi)}=\mathrm{i} \tau_{x}$

Observe that $\tau_{y}$ is delayed $\pi / 2$ with regard to $\tau_{x}$. However, in the present problem (type c), where the initial angular velocity vector (and the angular momentum) is oriented almost perpendicular to the axisymmetrical axis, the interesting case is $\theta \cong \pi / 2,(\theta=$ $\pi / 2-\delta$ ), and therefore

$\cos \theta=\cos \left(\frac{\pi}{2}-\delta\right)=\cos \frac{\pi}{2} \cos \delta+\sin \frac{\pi}{2} \sin \delta \cong \delta$

showing that the position of the axes are

1) $\tau_{z} \cong-\mathrm{i} \sin \theta e^{\mathrm{i} \psi} \cong-\mathrm{i} e^{\mathrm{i} \psi}$ (unity radius circumference)

2) $\tau_{x} \cong e^{\mathrm{i} t / r}(\cos \varphi+\delta \mathrm{i} \sin \varphi) \cong e^{\mathrm{i} t / r} \cos \varphi$, (spiral, circumference with radius $\cos \varphi(t))$

3) $\tau_{y} \cong e^{\mathrm{i} \psi}(-\sin \varphi+\delta \mathrm{i} \cos \varphi) \cong-e^{\mathrm{i} \psi} \sin \varphi$, (spiral, circumference with radius $\sin \varphi(t))$.

Observe that $\tau_{y}$ is delayed $\pi / 2$ with regard to $\tau_{x}$. Due to the presence of the sphere body itself, the axes $x$ and $y$ can be seen by the observer only when their $Z$ coordinate is $Z>0$

$Z_{x}=\sin \theta \sin \varphi \simeq \sin \varphi>0$

$Z_{y}=\sin \theta \cos \varphi \simeq \cos \varphi>0$

The distances of a point $\tau_{x}$ or $\tau_{y}$ in the spiral motion to the origin, $r_{x}, r_{y}$, are given, respectively, by

$r_{x}=\left|\tau_{x}\right|=\left(\cos ^{2} \varphi+\sin ^{2} \varphi \cos ^{2} \theta\right)^{1 / 2} \cong|\cos \varphi(t)|$

$r_{y}=\left|\tau_{y}\right|=\left(\sin ^{2} \varphi+\cos ^{2} \varphi \cos ^{2} \theta\right)^{1 / 2}=|\sin \varphi(t)|$

In Fig. 5 the observation windows for the different axes are shown superimposed. It can be deduced that the axes show up alternatively, as explained. Let us start at $\varphi=0$ (assuming appropriate initial conditions). The $y^{+}$axis appears at the centre of the axes $\left(r_{y} \cong 0\right) r_{y}$ growing monotonously towards the sphere contour rim $\left(r_{y}=1\right)$, and $\tau_{y}$ following a growing spiral, whilst the $x^{+}$ axis follows an opposite trajectory, from the $\operatorname{rim}\left(r_{x}=1\right)$ towards the centre $\left(r_{x}=0\right), \tau_{x}$ describing a decreasing spiral converging towards the origin when $\varphi=\pi / 2$, as axis $y^{+}$disappears by the sphere contour $\operatorname{rim}\left(y^{+}=1\right)$.

Then, $x^{+}$describes a spiral growing from $r_{x}=0$ at $\varphi=\pi / 2$ monotonously till reaching $r_{x}=1$ when $\varphi=\pi$. Simultaneously, axis $y^{-}$appears by the sphere contour rim, moving towards the origin. Afterwards $x^{+}$moves

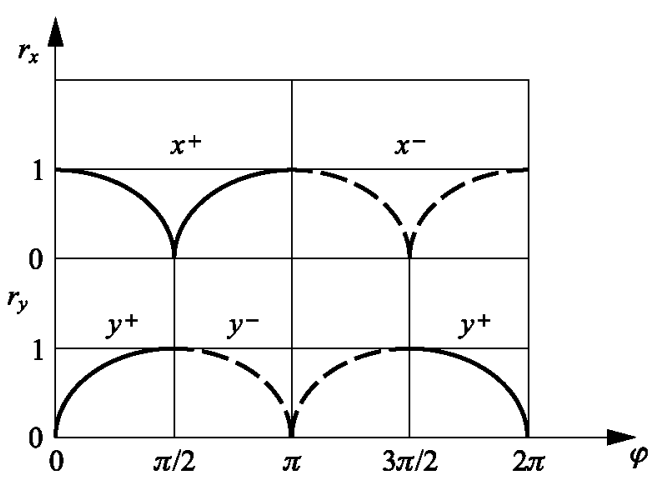

Fig. 5 Sketch of the variation of pole radial distances, $r_{x}$ and $r_{y}$, with the body spin angle $\varphi$. The observation windows are limited at the points where $z_{x}$ or $z_{y}$ are positive. Labels $x^{+}, y^{+}, x^{-}, y^{-}$, indicate intervals of $\varphi$ where these axes can be seen from $Z^{+}$ position

beyond the sphere contour rim placing itself in the back side of the sphere, disappearing of the field of view.

In the interval between $\varphi=\pi$ and $\varphi=3 \pi / 2$ the poles $y^{-}$and $x^{-}$repeat what the $y^{+}$and $x^{+}$poles performed in the interval $[0, \pi / 2]$, respectively. In summary, the sequence of appearance $y^{+}, x^{+}, y^{-}, x^{-}$is repeated in a cyclic way, in the case that $\varphi$ angle increases (and vice versa).

\section{Experimental Images}

It has been shown in the previous section that, when $\theta \cong \pi / 2$, the apparent motion of the each one of the principal inertia axes when looked at from $Z$-axis is a spiral with origin at the centre of the sphere and ending at the sphere contour (and vice versa), which is performed in a given sequence, one axis pole after the other. This sequence of axes is established in such a way that the centre of the spiral is occupied successively by the axis poles contiguous in the transversal plane, e.g. $x^{+}, y^{+}, x^{-}, y^{-}$. The theoretical model presented in "Motion of the Poles of the Principal Axes" was developed after a careful analysis and exploration of the video images obtained during the APIS experiment, with the idea of explaining, at least quantitatively, the curious behaviour that shows up in the video images. An example is shown in Fig. 6 where the growing spiral motion of the inertia axis pole ( $x^{-}$axis labelled with a GR sticker) can be followed in the sequence shown. A yellow star is superimposed on the video image to help in following the pole position. Sequences like that inspired the development of the model, and stressed the effort in the experiment analysis part of the work on determining the motion of the principal axes. 

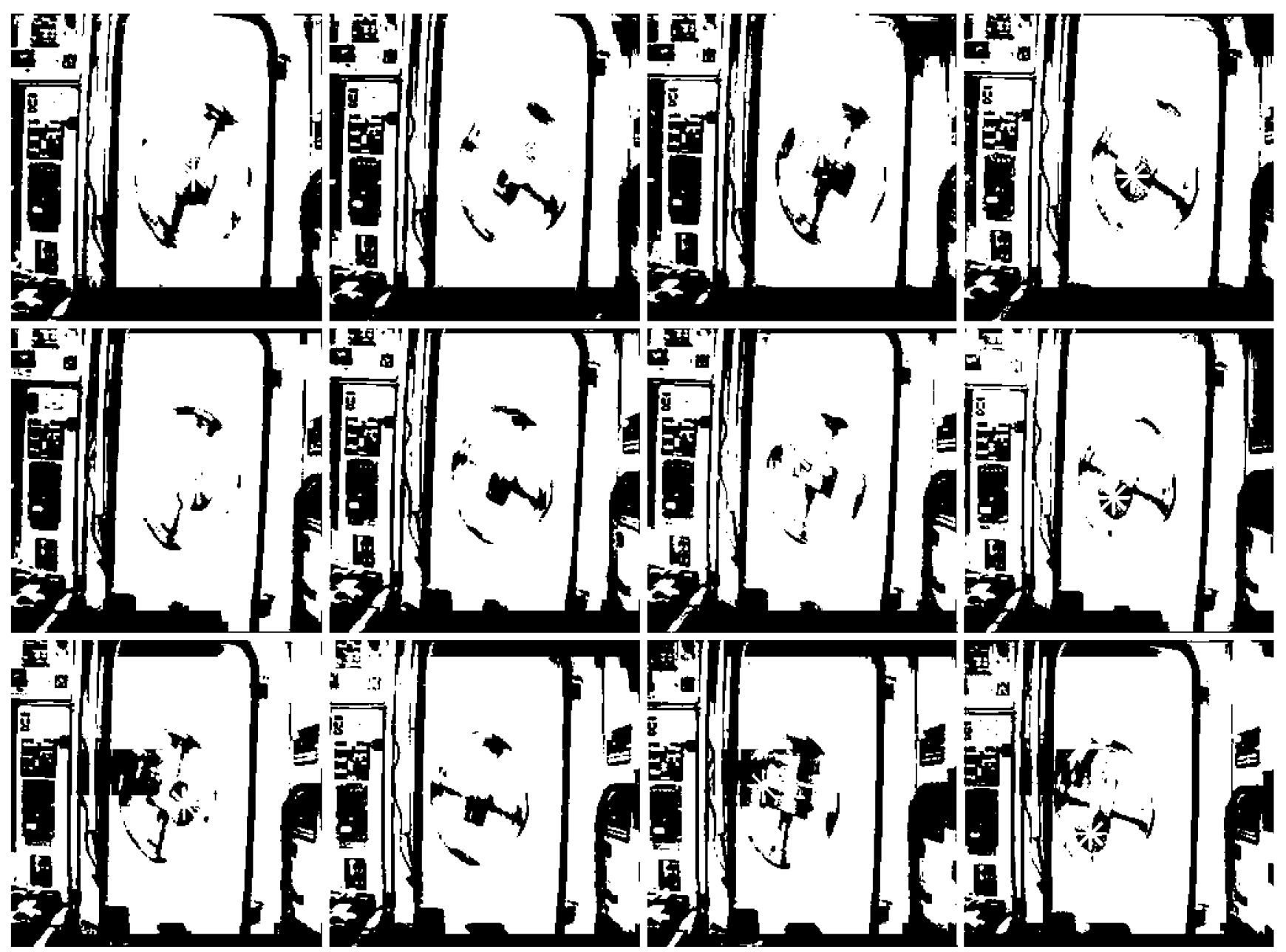

Fig. 6 Sequence of video images. Frames 23310 to 23320 . Each video image contains two consecutive frames. Time elapsed from left to right and from top to bottom. The superimposed yellow star indicates the position of the $x^{-}$(GR) principal inertia axis. It is spiraling out counter clockwise. The disks are placed together at the center
Furthermore, in the video images it can be observed that, just by chance, some kind of synchronism exists between the rotation rate and the image rate, in such a way that after four frames the original angular position of the pole with regard to the centre of the sphere is almost recovered, as is also the case for the angular position of the rotation of the sphere around the $x^{-}$ axis (observe the angular position of the body inside the sphere, which is quite similar in the pictures in each column).

The idea was to determine the position of the principal axis poles (which are easily detected thanks to the stickers) with regard to the sphere centre, where the centre of mass is placed. In each picture the sphere centre is determined by selecting four points evenly distributed along the sphere contour and obtaining the mean value. The sphere radius is obtained as the mean value of the distance of the four points to the centre. In such a way a circumference can be fitted to the sphere contour (see Fig. 7), the size of the sphere in the picture determines the scale and the dimensionless positions of the pole centre, and its coordinates with regard to the centre were obtained. It should be mentioned that, as the picture format produces a non-isotropic image, an aspect ratio correction was also needed. The spherical shape, selected on aerodynamic grounds, proved to be itself a very good choice for image processing reasons too, helping to greatly reduce the image analysis effort.

The results obtained for the apparent 2D position and apparent radius in a typical sequence are shown in Fig. 8. The 2D trajectory could be considered as a spiral, but with its centre displaced from the sphere centre. In the apparent radius evolution, several bumps are observed, that are not compatible with a spiral evolution, where the radius should vary in a monotonous way. This consideration leads to the conclusion that the 


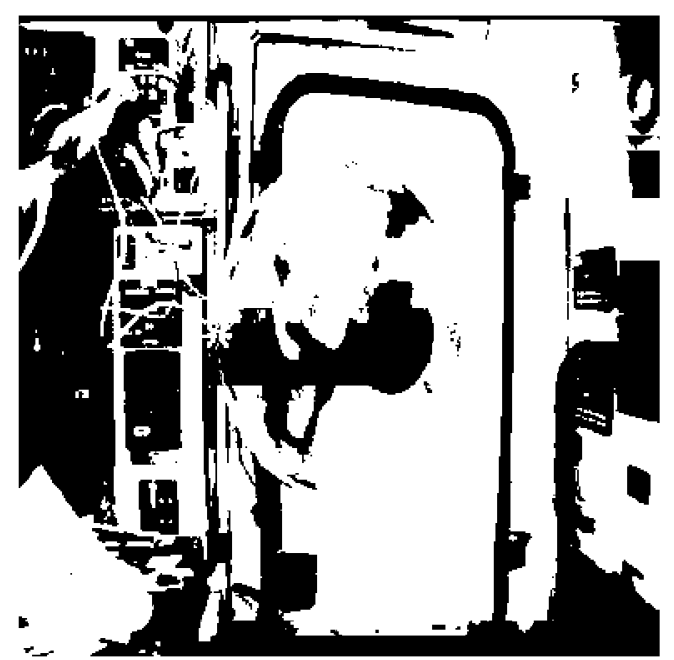

Fig. 7 Image analysis: determination of the sphere radius, centre and pole position. The disks are placed together at the center

camera line-of-sight was not quite aligned with the angular momentum axis, which the axis should move around. In consequence, a 3D coordinate rotation transformation was employed in order to change the
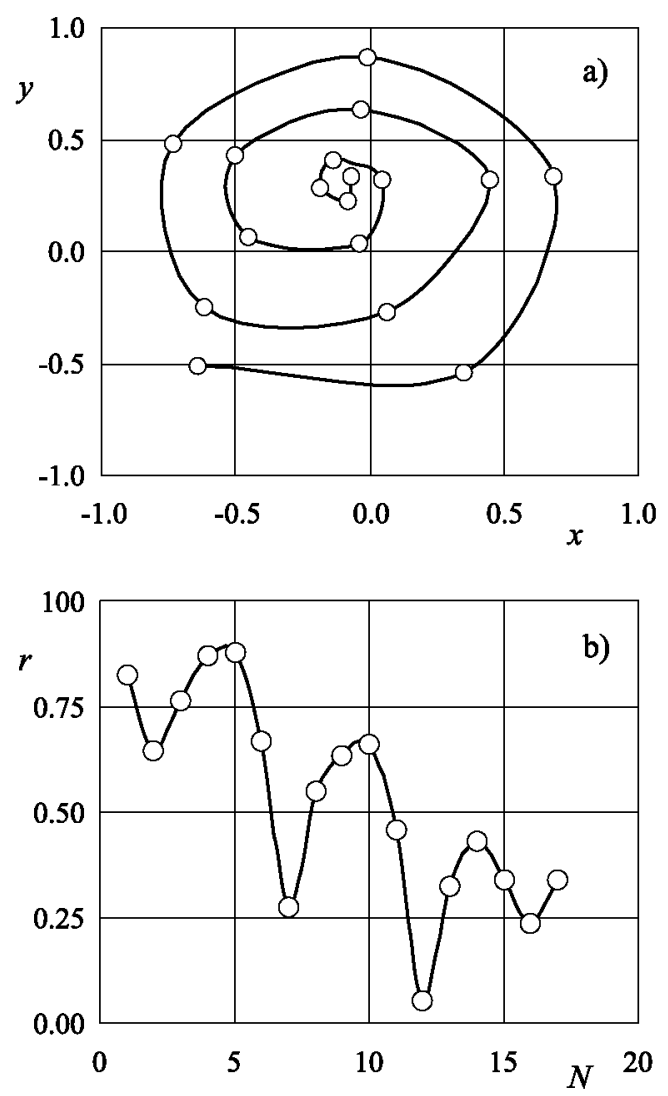

Fig. 8 WG $\left(x^{+}\right)$pole position evolution with time. a Apparent 2D position, $(x, y)$; b $r$ : Apparent radius, $N$ : frame number observation point-of-view of the camera. We were able to implement it thanks to that the shape of the object was known (a sphere) so that the three coordinates of the pole position on the sphere surface could be determined from its $2 \mathrm{D}$ coordinates in the $X-Y$ plane projection. This transformation could not have been performed if the shape of the object had been unknown (as it could be the case of an asteroid). Furthermore and fortunately, the sphere is the shape that involves the simplest transformations.

Other phenomena leading to this weaving variation of radius, like small differences among the transversal moments of inertia, were analyzed but finally not implemented due to the difficulty to identify the relevant parameters involved, taking into account that the previous correction is just an approximation. The effect of small differences among transversal moments of inertia is discussed in the next section.

\section{Experimental Results}

By performing the abovementioned three dimensional coordinate transformation, which puts the centre of the spiral at the centre of the sphere on the projection on the $\mathrm{X}-\mathrm{Y}$ plane, both the projection of the axis pole, "corrected 2D position", and the new distance to the centre, denoted "corrected radius", can be obtained (see Fig. 9). It can be shown that the shape of the radial variation is closer to have a monotonous slope than in the case of "apparent" results. But a completely monotonous shape could not be obtained by this method because there are more circumstances that should be taken into account, as for instance, that the point of view of the camera changed along the recording of the experiment and therefore the "corrected" data could not be completely corrected. The above-mentioned process has been applied to two experimental runs:

1. With the maximum transversal inertia (disks placed apart 100\%). Video frames 8:47 to 8:54 (Fig. 10a). Numbers are in seconds.

2. With minimum transversal inertia (disks placed together at the sphere centre). Video frames 15:30 to 15:43 (Fig. 10b).

In the first case an evolution close to the one expected from the theoretical model can be observed. First the GW $\left(x^{+}\right)$pole follows a spiral motion towards the sphere centre, reaches the centre, and then spirals out towards the rim of the sphere image. When it is close 

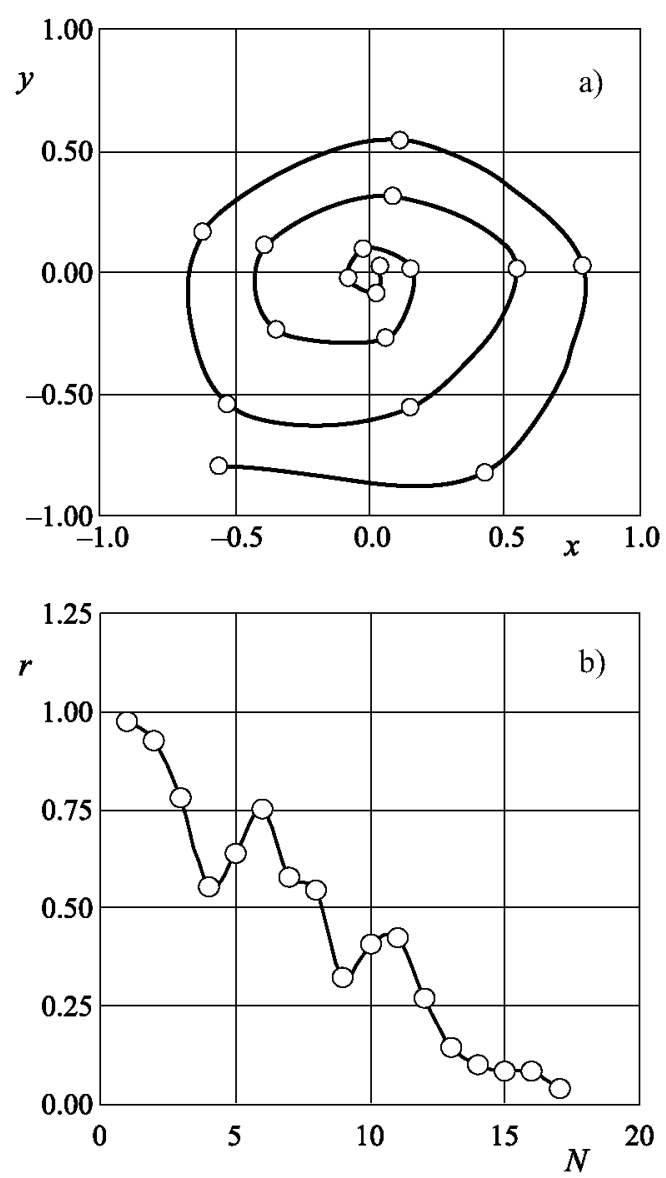

Fig. 9 WG $\left(x^{+}\right)$pole position evolution with time (closing spiral). a Corrected $2 \mathrm{D}$ position $(x, y) ; \mathbf{b} r$ : Corrected radius, $N$ : frame number

to the rim the $\mathrm{KR}\left(y^{-}\right)$pole enters into the image, spiralling towards the sphere centre, repeating the process carried out by the $\mathrm{GW}\left(x^{+}\right)$.

It is shown in the video images that the same process is repeated afterwards by poles $x^{-}$and $y^{+}$following the expected sequence. The repetition of the sequences confirms that the effect of air resistance is not relevant in the time scale of the experiment.

In the second run the following sequence (which at first glance looks similar to the one previously discussed) is observed: the first pole that appears, GR $\left(x^{-}\right)$, describes a convergent spiral, almost reaches the sphere centre and starts to spiral out towards the sphere image rim, but instead of performing the pole exchange, the same pole comes back spiralling towards the sphere centre again. Then the pole describes a nominal sequence (spiralling in, pass by the centre, spiralling out) and exchanges evolution with the other pole $\mathrm{KR}\left(y^{-}\right)$.

But the anomaly continues: the next poles passes observed are GR $\left(x^{-}\right)$and $\mathrm{KR}\left(y^{-}\right)$instead of the
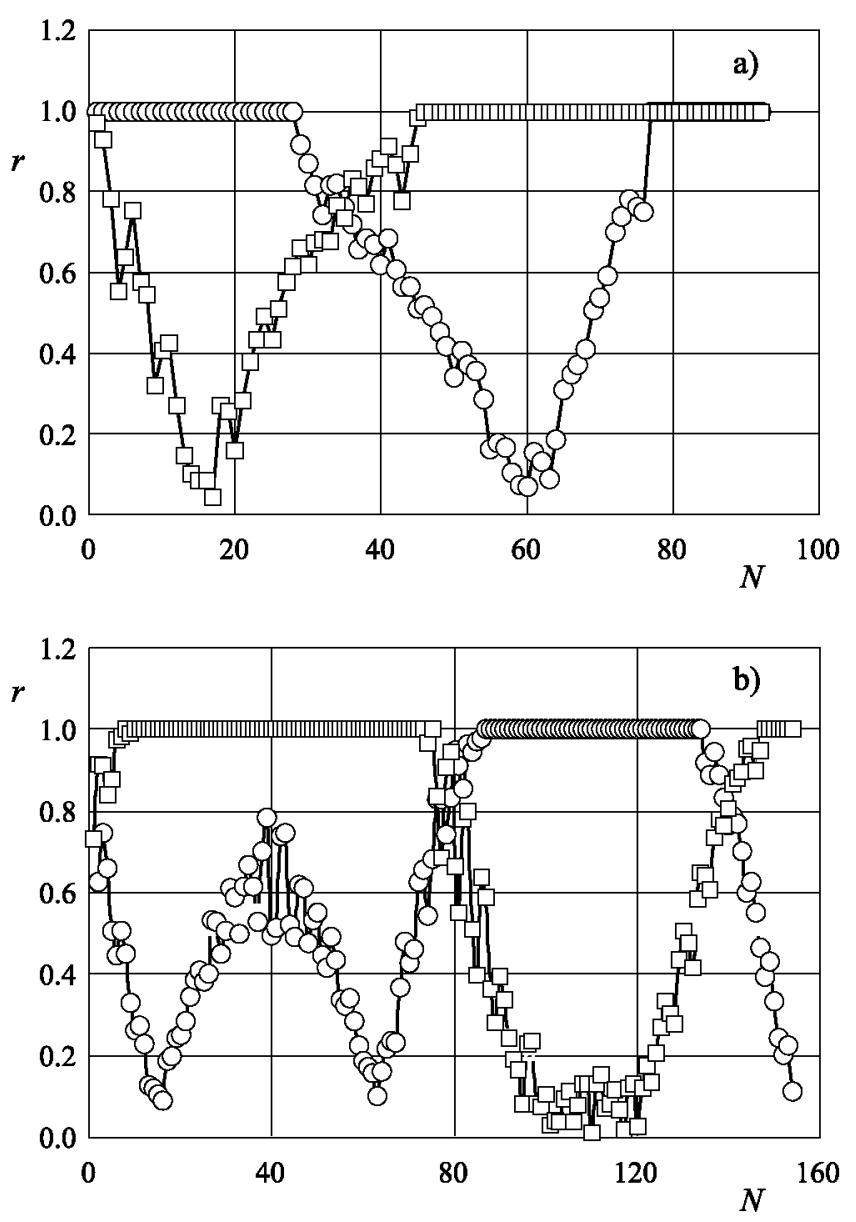

Fig. 10 Pole position evolution with time. $r$ : Corrected radius, $N$ : frame number. Body with a maximum, and $\mathbf{b}$ minimum, transversal inertia. Symbol key: a squares, $\mathrm{GW}_{-} \boldsymbol{x}^{+}$, circles, $\mathrm{KR}-y^{-}$. b squares, KR- $y^{-}$, circles, GR- $x^{-}$

expected ones $\left(x^{+}, y^{+}\right)$that do not show up. To explain the behaviour shown in run 2 a more sophisticated reasoning is needed. It has been deduced from numerical simulation that a change in the $\omega_{z}$ rotation speed component sign is needed in order to explain the return of the GR $\left(x^{-}\right)$pole. Moreover, the reason for this $\omega_{z}$ sign change could be that the body is not exactly axisymmetrical (as assumed) due to the existence of a small difference among the moments of inertia $A$ and $B$ of the transversal axes, $x, y$, respectively. However, to clarify this point, more accurate determination of the inertia properties of the body should be available.

\section{Conclusions}

The free rotational motion of a solid can be only studied in the gravity free environment of an orbiting laboratory as the International Space Station. 
As a result of the performance of the educational experiment APIS several sequences of video images of the motion of bodies with different inertia properties have been obtained. The external shape of the bodies is a sphere to reduce the aerodynamic torque produced by the fluid to just the friction acting on the body surface. Some sequences of these motions have been analyzed, focusing on one of the characteristics of the motion, namely the trajectory of the points of the sphere that represents the direction of the principal inertia axes (axis poles).

The sequences analyzed illustrate some very curious demonstrations of the classical solution: the sphere performs a sequence of rotations around the principal inertia axes perpendicular to the axisymmetry axis in a given order. One of these demonstrations can be easily explained by using the solutions of the Euler equations, but in the other case to find an explanation needs further study.
Acknowledgements The authors would like to thank the members of the student team (Borja Vera-Carrasco, Fernando Meseguer-Garrido, Cristina Montalvo-Martín, Félix Parra-Díaz, Miguel Angel Torralba and Violeta Medina-Andrés) for their effort, under the support of the IDR-UPM, and the astronaut Pedro Duque for their special collaboration and initiative.

\section{References}

Asphaug, E., Scheeres, D.J.: Deconstructing Castalia: evaluating a postimpact state. Icarus 139, 383-386 (1999)

Pravec, P., Harris, A.W., Michalowski, T.: Asteroid rotations. In: Bottke, W.F. Jr., et al. (eds.) Asteroids III, pp. 113-122. Univ. of Arizona, Tucson (2002)

Steel, D.I., McNaught, R.H., Garradd, G.J., Asher, D.J., Taylor, A.D.: Near-earth asteroid 1995 HM: a highlyelongated monolith rotating under tension? Planet. Space Sci. 45(9), 1091-1098 (1997)

Thomson, W.T.: Introduction to Space Dynamics. Dover Publications, New York (1986)

Wertz, J.R.: Spacecraft Attitude Determination and Control, p. 501. Kluwer Academic, Berlin (1978) 